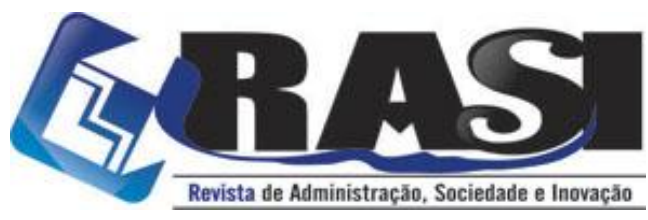

http://www.rasi.vr.uff.br

RASI, Volta Redonda/RJ, v. 6, n. 3, pp. 22-41, set./dez. 2020

\title{
Mensuração do Processo de Cocriação de Valor em Empresas do Setor Bancário Brasileiro.
}

Bruno Valente Zero (USP) - brunovzero@gmail.com

Ronaldo de Oliveira Santos Jhunior (USP) - ronaldojhr@usp.br

João Maurício Gama Boaventura (USP) - jboaventura@usp.br

\begin{abstract}
RESUMO
A realidade emergente pautada no maior acesso a informações, na convergência tecnológica, e na rápida evolução da internet como meio para comunicação e, também, realização de negócios, exige uma reavaliação do sistema tradicional de criação de valor centralizado na empresa. Este artigo se direciona para a ideia da cocriação de valor, na qual o valor não é mais definido dentro da empresa e apenas entregue ao consumidor, mas sim criado conjuntamente com stakeholders em um processo focado nos indivíduos e suas experiências. Dessa forma, o estudo busca ampliar conhecimento acerca da cocriação de valor em empresas brasileiras com base em informações empíricas, para tanto, o objetivo da pesquisa se traduz em mensurar a cocriação de valor em empresas do setor bancário brasileiro e fazer uma comparação do desempenho entre elas. Utiliza-se o modelo DART (diálogo, acesso, risco e transparência), proposto por Prahalad e Ramaswamy (2004), como base de análise dos dados obtidos no Índice de Sustentabilidade Empresarial BM\&F-ISE referente a 2017. Nos resultados, percebe-se o destaque de um dos bancos analisados, bem como o fato de que as empresas analisadas ainda precisam de maior foco no desenvolvimento de fatores relacionados a dimensão "acesso" do modelo utilizado.
\end{abstract}

PALAVRAS-CHAVES: Valor; Criação de valor; Cocriação de valor; Setor bancário.

\section{Measurement of the Value Co-Creation Process in Brazilian Banking Sector Companies.}

\begin{abstract}
The emerging reality based on greater access to information, technological convergence, and the rapid evolution of the internet as a means of communication and conducting business, requires a reevaluation of the traditional enterprise-centric value creation system. This article addresses the idea of value co-creation, in which value is no longer defined within the company and only delivered to the consumer, but rather created jointly with stakeholders in a process focused on individuals and their experiences. Thus, the study seeks to expand knowledge about value co-creation in Brazilian companies based on empirical information. Therefore, the objective of the research is to measure value co-creation in companies in the Brazilian banking sector and to compare performance between them. The DART model (dialogue, access, risk, and transparency) proposed by Prahalad e Ramaswamy (2004) is used as the basis for the analysis of data obtained from the Corporate Sustainability Index (BM\&F-ISE) of 2017. The results show the highlight of one of the analyzed banks, as well as the fact that the analyzed companies still need a greater focus on the development of factors related to the "access" dimension of the model used.
\end{abstract}

KEY WORDS: Value; Value creation; Value co-creation; Bank sector.

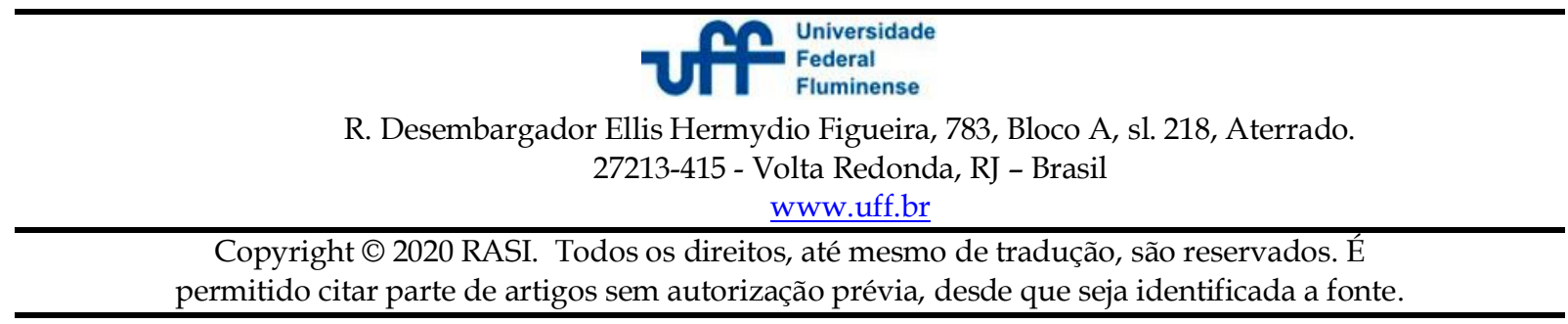




\section{Mensuração do Processo de Cocriação de Valor em Empresas do Setor Bancário Brasileiro.}

\section{Introdução}

Ao longo das últimas duas décadas, diversas formas de conhecimento e informação tornaram-se acessíveis, alterando processos tradicionais de negócios e maneiras que empresas lidam com inovação (Pater, 2009). Fenômenos como a desregulamentação, a convergência tecnológica, e a rápida evolução da internet redefiniram os limites da indústria e o desenvolvimento de produtos. Dessa forma, observa-se que o mercado se torna inseparável do processo de criação de valor e o converte em um fórum onde consumidores, firma, comunidades e redes dialogam e trocam conhecimento (Silva, \& Villan, 2018).

Para Prahalad e Ramaswamy (2004), essa realidade emergente exige reavaliação do sistema tradicional de criação de valor centralizado na empresa. A resposta, para os autores, está na ideia da cocriação de valor, ou seja, o valor não é mais definido dentro da empresa e apenas entregue ao consumidor, mas sim criado conjuntamente com este em um processo focado nos indivíduos e suas experiências. A cocriação se torna, dessa forma, a base da definição de valor no atual contexto do mercado e pode servir de subsídio para o futuro da organização (Mangini et al., 2018).

A ideia de cocriação de valor vem se tornando um tema de relevância crescente na literatura recente de administração (Kujala, Lehtimäki, \& Myllykangas, 2017) desde os estudos de Vargo e Lusch (2004) e seus incentivos no desenvolvimento da temática. O tópico da cocriação de valor despertou o interesse de pesquisadores e praticantes como um conceito que visa descrever a colaboração entre múltiplos stakeholders (Prahalad, \& Ramaswamy, 2000), apesar do foco ainda fortemente direcionado às relações com o stakeholder consumidor, seguindo influências de estudos voltados para a área de marketing (Vargo, \& Lusch, 2004; Vargo, Maglio, \& Akaka, 2008; Ranjan, \& Read, 2014). No entanto, há também o argumento acerca do conceito de cocriação de valor indo além de apenas dar ao cliente um papel mais proativo (Alves, Fernandes, \& Raposo, 2016; Kujala, Lehtimäki, \& Myllykangas, 2017). Assim, o conceito pode ser descrito como um processo mais participativo de criação de valor, no qual organizações e stakeholders diversos são capazes de gerar e desenvolver valor e significado (Alves, Fernandes, \& Raposo, 2016; Ind, \& Coates 2013).

Randall, Gravier e Prybutok (2011) sustentam que a operacionalização da cocriação, suasdimensõeseseutesteempíricoaindarepresentamumalacunanessaáreadepesquisa. Dito isso, observa-se que apenas um artigo do tema cocriação foi divulgado em periódicos do Brasil entre 1999 e 2011 (Ribeiro, \& Costa 2016), uma lacuna temporal de mais de uma década. Nessa perspectiva, a presente pesquisa reconhece a ainda incipiente literatura de cocriação de valor no contexto brasileiro, bem como a inexistência de uma análise de correlação entre a cocriação e outras variáveis de desempenho no mesmo cenário em setores específicos e procura contribuir para a ampliação do conhecimento acerca da referida temática no Brasil. Dessa forma, o estudo busca compreender como ocorre a operacionalização da cocriação de valor em empresas brasileiras salientando as relações desta com o desempenho empresarial.

Para a realização desta pesquisa foi selecionado o setor bancário brasileiro devido ao seu reconhecido elevado padrão no que se refere a desempenho e competitividade, o que faz deste um setor interessante para o desenvolvimento de estudos relacionados à análise de stakeholders (Rudzevicius et al., 2018). Além disso, a participação das empresas brasileiras 
do setor bancário no ISE é mais evidente quando em comparação com empresas de outros setores.

A pesquisa objetiva, portanto, mensurar a operacionalização da cocriação de valor em empresas do setor bancário brasileiro e fazer uma comparação do desempenho entre elas. O modelo DART, proposto por Prahalad e Ramaswamy (2004), foi utilizado como base para análise dos dados obtidos no Índice de Sustentabilidade Empresarial BM \& F-ISE, fonte das informações utilizadas na pesquisa. Para tanto, procurou-se rever a literatura pertinente ao objeto de estudo para em seguida realizar uma pesquisa empírica utilizando o modelo mencionado.

Para a apresentação do estudo, além desta introdução, o artigo está organizado em outras seções. A seguir, discutimos o referencial teórico abordando brevemente conceitos relacionados a ideia da criação e cocriação de valor, bem como do modelo base para o desenvolvimento da pesquisa. Posteriormente, encontram-se expostos os procedimentos metodológicos seguidos na investigação, a análise e discussão dos resultados e, por fim, as considerações finais.

\section{Referencial Teórico}

A participação de consumidores na criação de valor começou a ser abordada por Eric von Hippel (1986), através da user innovation. Essa metodologia consistia na identificação, por parte da empresa, de consumidores líderes (Lead Users), que adaptavam seus produtos de acordo com suas necessidades. Esses clientes identificavam os problemas nos produtos antes da maioria dos consumidores e, dessa forma, suas ideias podiam ser aplicadas no âmbito produtivo. Mais adiante, o termo de cocriação foi primeiramente explorado por Prahalad e Ramaswamy (2000). Segundo os autores, os consumidores cocriam e extra em valor do negócio. Assim, passam a ser colaboradores, co-desenvolvedores e competidores no mercado.

Em 2004 o tema foi novamente desenvolvido pelos mesmos autores no livro The Future of Competition, quando o conceito se torna amplamente difundido. Os autores sustentam que os consumidores buscam a liberdade de escolha para interagir com empresas através de suas experiências, isto é, desejam refletir sua visão de valor e utilizar sua própria linguagem. O conceito também foi desenvolvido por Stephen L. Vargo e Robert F. Lusch (2004), os quais argumentam que a lógica dominante focada em recursos tangíveis, valor "embutido" e transações é substituída por uma nova perspectiva de intangibilidade, cocriação de valor e relacionamento.

Em 2010 Francis Gouliart e Ventak Ramaswamy pubicaram o livro The Power of Cocreation: Build it with them to boost growth, productivity and profits. Os autores defendem que a cocriação envolve redefinir a forma como a organização engaja clientes, funcionários, fornecedores e parceiros, e outros. Dessa forma, o conceito extrapola o âmbito dos consumidores para ser aplicado também em relação a todos os stakeholders.

Para Kujala, Lehtimäki e Myllykangas (2017), o gerenciamento de ativos intangíveis se torna um fator-chave de sucesso e o valor intangível é criado em relacionamentos organizacionais. Para que o negócio mantenha seu valor aos olhos dos clientes e outros stakeholders, o negócio e sua lógica precisam mudar (Kujala, Lehtimäki, \& Myllykangas, 2017). Para Parmar et al. (2010), a economia global está intimamente interconectada por meio de mercados financeiros abertos e tecnologia da informação e comunicação e, portanto, é necessária uma nova narrativa para a criação de valor nos negócios.

\subsection{Conceito de Valor}


Destaca-se primeiramente que as pessoas fazem escolhas tendo por referência seus valores pessoais, os quais são representações cognitivas e transformações de suas necessidades humanas (Rokeach, 1973). Ainda para o autor, as pessoas têm nos seus valores, parâmetros que guiam processos conscientes e inconscientes que justificam e racionalizam suas ações, pensamentos e julgamentos. Overby (2001) apresenta a taxonomia das teorias de valor, relacionando a teoria, sua definição e os artigos que a abordaram, representados no Quadro 1 abaixo.

\begin{tabular}{|c|c|c|}
\hline Teoria & Definicão de valor & Artigos \\
\hline $\begin{array}{l}\text { Transação } \\
\text { específica }\end{array}$ & $\begin{array}{l}\text { Valor é a economia obtida em uma transação } \\
\text { (preço de referência menos preço dado). }\end{array}$ & $\begin{array}{l}\text { Szybillo, \& Jacoby (1974); } \\
\text { Berkovitz, \& Walton (1980); } \\
\text { Urbany et al. (1988) }\end{array}$ \\
\hline $\begin{array}{c}\text { Qualidade } \\
\text { ajustada ao } \\
\text { preço }\end{array}$ & $\begin{array}{l}\text { Valor é a quantidade condicionada ao preço } \\
\text { (qualidade/preço). }\end{array}$ & $\begin{array}{l}\text { Monroe (1990); Tellis, \& Gaeth } \\
\text { (1990); Dodds, Monroe, \& Grewel } \\
\text { (1991); Gale, \& Wood (1994); } \\
\text { Bolton, \& Drew (1991) }\end{array}$ \\
\hline $\begin{array}{l}\text { Orientado para } \\
\text { utilidade }\end{array}$ & $\begin{array}{c}\text { O valor depende da utilidade do produto ou da } \\
\text { utilidade condicional ao sacrifício feito } \\
\text { (Utilidade de transação + utilidade de } \\
\text { aquisição). }\end{array}$ & $\begin{array}{c}\text { Krishnamurti (1982); Thaler (1985); } \\
\text { Hauser, \& Urban (1986); Zeithaml } \\
\text { (1988) }\end{array}$ \\
\hline Experiencial & $\begin{array}{l}\text { Valor é uma experiência interativa ou uma } \\
\text { noção subjetiva derivada da experiência. }\end{array}$ & $\begin{array}{c}\text { Holbrook, \& Hirschman (1982); } \\
\text { Holbrook, \& Corfman (1985); } \\
\text { Holbrook (1994); Aurier, \& Ervard } \\
\text { (1997) Aurier, Evrard, \& N'Goala } \\
\text { (1998, 2000), Evrard, \& Aurier } \\
\text { (1995, 1996) }\end{array}$ \\
\hline
\end{tabular}

Fonte: Overby (2001)

A teoria da transação específica de valor enfatiza a perspectiva econômica. Para Richins (1994), a literatura econômica sobre valor sempre atrela esse conceito no contexto de troca, ou seja, quanto os consumidores estão dispostos a pagar. No entanto, para o autor, o valor de posse é mais adequado, que se refere ao valor de uso do produto e não apenas a seu valor econômico.

Na perspectiva da qualidade ajustada ao preço, Dodds et al. (1991) afirma que o valor é um trade-off cognitivo entre qualidade percebida e sacrifício. Fornell et al. (1996), por sua vez, sustenta que valor é o nível de percepção da qualidade de um produto relativo ao preço pago, definição também compartilhada por Monroe (1990). Na visão de Gale e Wood (1994, p. 14) a definição de valor seria "a qualidade percebida pelo mercado, ajustada ao preço relativo de seu produto".

Já na ótica da orientação para utilidade, Zeithaml (1988) define valor como a avaliação geral da utilidade de um produto baseado na percepção do que é dado em troca do que é recebido. Woodruff e Gardial (1996) compartilhavam essa perspectiva, afirmando o valor como a percepção do consumidor sobre o que ele deseja que aconteça em uma específica situação de uso, com a ajuda de algum produto ou oferta de serviço, no sentido de alcançar alguma proposta e meta.

Por último, na vertente experiencial de conceito de valor, Holbrook (1999) afirma que a experiência de valor para o consumidor envolve quatro dimensões: (i) interatividade, (ii) relatividade, (iii) preferência e (iv) experiência de consumo. O valor é relativo porque depende das características de um objeto associado ao envolvimento do sujeito. É relativo por ser resultado de avaliações de clientes em diferentes situações. Na dimensão (iii) Holbrook 
(1999) sustenta que o valor envolve julgamentos de preferência feitos pelos consumidores, com o afeto, opinião, predisposição e avaliação. Já na (iv) experiência de consumo o autor defende que o valor está na relação de o produto e a utilização depois de ser adquirido.

\subsection{Criação de Valor na Perspectiva Tradicional}

O pensamento do negócio tradicional começa com a premissa que a empresa cria valor (Prahalad, \& Ramaswamy, 2004a). A empresa de forma autônoma determina o valor que irá oferecer por meio da escolha de produtos e serviços. O consumidor representa a demanda para a oferta e as implicações para o negócio seguem essas premissas. Assim, a firma necessita de uma interface com consumidores para trocar seus produtos e serviços e desenvolve abordagens múltiplas pra extrair esse valor: aumentando a variedade de oferta, entregando de forma eficiente os serviços e produtos e customizando para os consumidores individualmente.

Essas premissas e implicações se manifestam nas perspectivas e práticas das firmas no sistema industrial. Dessa forma os gestores focam no canal de valor que captura o fluxo de produtos e serviços por meio de operações que a firma controla ou possui influência. Nesse cenário, o cliente tem pouco ou nenhum papel na criação de valor.

$\mathrm{Na}$ visão de Ramaswamy e Gouillart (2010) as empresas tradicionais estabelecem "pontos de contato" para com clientes, fornecedores, parceiros e funcionários. No entanto, esses pontos são apenas na perspectiva da empresa, além de serem escassos e breves. Os clientes são unicamente apresentados com o design oferecido pela empresa, ou seja, não participam do processo. Ainda para os autores, esse paradigma tradicional atendeu o mercado por muitos anos, mas passa por processo de obsolescência.

Figura 1 - A Concepção Tradicional de Mercado

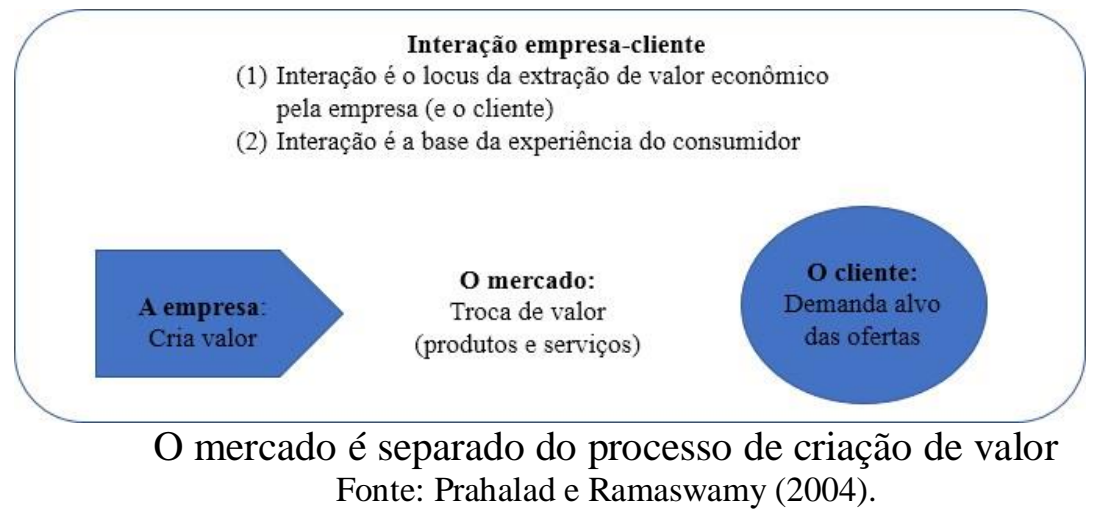

Segundo Prahalad e Ramaswamy (2004), o sistema tradicional de mercado era centralizado na empresa. Os consumidores eram passivos já que significam somente o público alvo da indústria. As firmas focavam no lócus de interação e de extração de valor econômico e o mercado tinha funções diferentes para as empresas e para os consumidores. Dessa forma, o mercado era separado do processo de criação de valor, como mostra a Figura 1 acima.

\subsection{Cocriação de Valor}

Na visão de Franco (2012), um antecedente notório do conceito de cocriação foi o método Lead User, proposto por Eric Von Hippel (1986). Lead Users são pessoas que têm explorado de formas inovadoras o desenvolvimento de ideias e que estão interessados em compartilhá-las (Sanders, \& Stappers 2008). No cenário mercadológico contemporâneo, 
observa-se a necessidade de atualização no relacionamento com stakeholders. O crescimento da internet, das mídias sociais e os avanços em mobile fazem com que indivíduos ligados em rede ao redor do mundo não sejam mais receptores passivos, dispensados de instruções e assistência para serem participantes ativos, colaboradores em processos de criação de valor e cocriadores de soluções com empresas de diversos nichos (Ramaswamy, \& Gouillart, 2010).

Conforme mencionado, o termo "cocriação" foi primeiramente utilizado por Prahala de Ramaswamy no artigo "Co-Opting Customer Competence" publicado no ano 2000. Na visão dos autores, os clientes ultrapassaram seus papéis tradicionais para se tornar cocriadores, assim como consumidores de valor. Segundo os autores o cliente é o agente que está transformando de forma mais intensa o sistema industrial como é conhecido.

De acordo com Prahalad e Ramaswamy (2000), a mudança mais significativa da cocriação tem sido uma reorientação da função do consumidor, de isolado para conectado, de não informado para informado e de passivo para ativo. A internet possibilitou um diálogo ativo e explícito entre clientes e fabricantes de produtos e serviços, sendo que esse diálogo não é mais controlado pelas organizações. Essa reorientação é expressa no processo a seguir.

Figura 2 - O Novo Quadro de Referência para Criação de valor

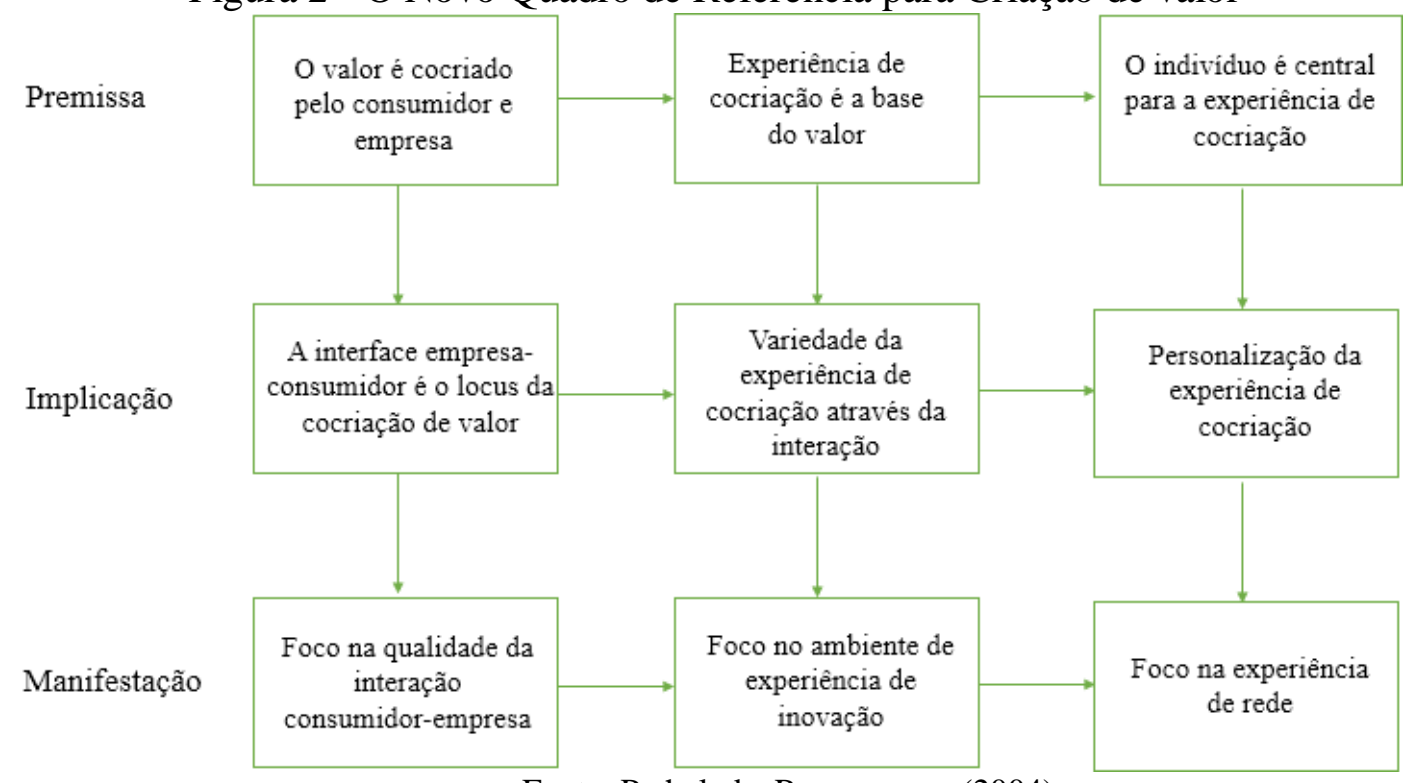

Fonte: Prahalad e Ramaswamy (2004)

O novo processo começa com a premissa de que o consumidor e a empresa cocriam valor, sendo que essa experiência se torna a base do valor. Dessa forma, o processo de criação de valor centraliza no indivíduo e sua experiência de cocriação. Isso implica na mudança da fonte de criação de valor, que passa a ser a interface empresa-consumidor.

Como existem diferentes tipos de interações, é criada uma variedade de experiências de cocriação, gerando uma personalização de cada uma. Esse processo se manifesta no foco, na qualidade da interação, na experiência de inovação e na experiência de rede (Prahalad, \& Ramaswamy, 2004).

Entrando na abordagem conceitual, Ramaswamy e Goulliart (2010) sustentam que a cocriação representa o desenvolvimento de novos modos de engajamento para que indivíduos possam inserir-se na cadeia de valor da organização. Os autores sustentam que o futuro das empresas depende da cocriação, e que marketing tradicional em que as empresas criam e as 
pessoas consomem estaria se extinguindo. Zwass (2010) define cocriação como participação dos consumidores, juntamente com os produtores, na criação de valor no mercado, podendo ser iniciada tanto pelas empresas como pelos consumidores. Em outra perspectiva, segundo Ramaswamy e Ozcan (2014), a cocriação seria a junção do desenvolvimento de valor com os stakeholders, este potencializado por meio de plataformas de engajamento e atualizado em domínios de experiências, expandindo riqueza, saúde e bem-estar. Choi e Burnes (2013), por sua vez, argumentam que o relacionamento mais próximo entre os stakeholders leva à cocriação de valor, com grupos distintos revisando diferentes formas de valor, o que aprimora o relacionamento entre os stakeholders.

No que se refere a estudos mais recentes, Tantalo e Priem (2016) introduzem a ideia da sinergia dos stakeholders, uma abordagem que busca redefinir a maneira como acadêmicos e praticantes pensam a criação de valor. Considerando a criação de valor como essencial para o sucesso estratégico organizacional, os autores argumentam que tal sinergia seria capaz de incrementar diferentes tipos de valor para grupos de interesse distintos simultaneamente. Para Alves, Fernandez e Raposo (2016), A Cocriação de valor não é apenas um conceito-chave em reflexões acerca do marketing de serviços e do gerenciamento de negócios, mas também é um termo capaz de descrever uma mudança na consideração da organização como um definidor de valor para um processo mais participativo no qual indivíduos e organizações em conjunto geram e desenvolvem significado.

Com base em Ramaswamy e Gouillart (2010), essa dinâmica faz com que se torne necessária uma nova forma de envolvimento dos stakeholders - clientes, funcionários, fornecedores, parceiros, governos, investidores, cidadãos e outros - para se criar valor. Assim, os stakeholders não são mais somente públicos passivos, mas sim uma parte ativa na relação com as empresas. Prahalad e Ramaswamy (2004) afirmam que na concepção emergente de mercado o foco se encontra na interação empresa-consumidor. A empresa e o consumidor são ambos colaboradores e competidores-colaboradores na cocriação de valor e competidores na extração de valor econômico.

\subsection{Modelo DART}

Ramaswamy e Ozcan (2014) sustentam que para desenvolver um método de cocriação de valor é importante começar com "blocos" de interações que facilitam experiências de cocriação entre as empresas e os clientes. Prahalad e Ramaswamy (2004) definem esses blocos como diálogo, acesso, benefícios-risco e transparência (DART), representados na Figura 3.

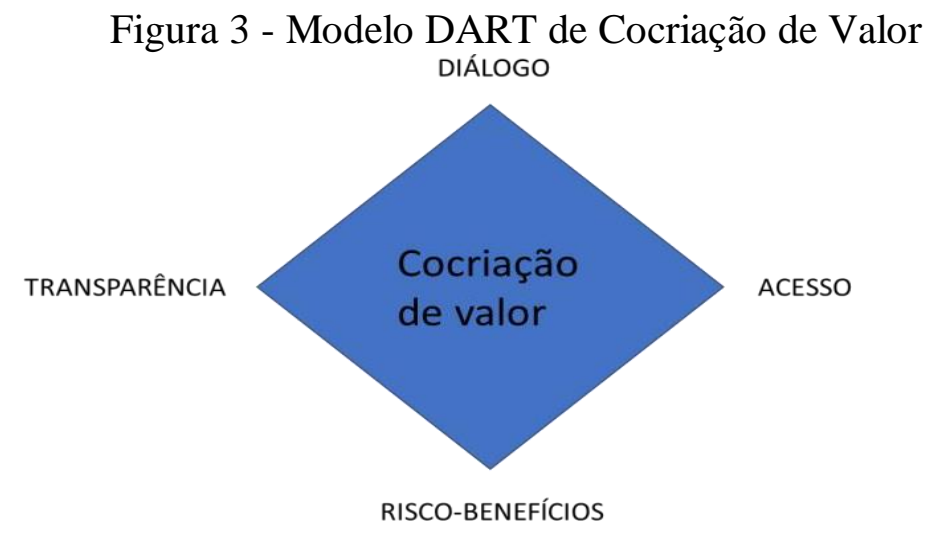

Fonte: Prahalad e Ramaswamy (2004) 
A primeira dimensão do modelo representa ou diálogo, elemento como chave na realização de trocas e relacionamentos bem-sucedidos entre as partes interessadas (Raelin, 2013; Chakraborty, \& Dobrzykowski, 2014). De acordo com Prahalad e Ramaswamy (2004) diálogo - é a interatividade, engajamento profundo e propensão a agir de ambos os lados. $\mathrm{O}$ diálogo auxilia as empresas a compreenderem os contextos culturais, sociais e emocionais das experiências de consumo na tentativa de fornecer conhecimentos para inovação. Além disso, implica em aprendizado e comunicação compartilhada igualmente para solução de problemas. Para os autores, o diálogo envolvido na cocriação possui três características principais:

- Foca em questões que interessam tanto o cliente como a empresa;

- Necessita de um fórum aonde o diálogo possa ocorrer;

- Requere regras de engajamento (explicitas ou implícitas) que promovem uma interação produtiva.

$\mathrm{O}$ acesso consiste na experimentação do produto ou serviço sem necessariamente ter a posse deste. A visão tradicional das empresas e suas cadeias de valor era criar e transferir a posse de seus produtos para os consumidores. No entanto, cada vez mais o objetivo dos clientes é o acesso a experiências e não a posse do produto. Risco refere-se a chance de prejudicar o consumidor. Na comunicação com os consumidores, o marketing foca quase totalmente em expor benefícios, ignorando os riscos. No entanto, é coerente assumir que como cocriadores do processo, os clientes vão insistir em saber não só informações como também metodologias apropriadas para acessar os riscos pessoais e sociais associados aos produtos. Transparência significa reduzir a assimetria de informações entre a empresa e o cliente. Empresas não devem mais negligenciar informações sobre preços, custos, e margens de lucro. Na medida em que informações sobre produtos, tecnologias e sistemas de negócios se tornam mais acessíveis, novos níveis de transparência são exigidos.

Em suma, para além do trabalho seminal apresentado, conceitua-se a ideia de cocriação de valor como o diálogo e a comunicação entre stakeholders; a capacidade dos stakeholders de acessar e compartilhar dados; a capacidade de monitorar benefícios-risco; e, finalmente, a transparência entre os stakeholders, eliminando possíveis barreiras de informação (Oliveira, Damacena, \& Brambilla, 2014; Johns, \& Davey, 2019). Dessa forma, para Ramaswamy e Ozcan (2014), a cocriação de valor expande a criação tradicional de valor em três vertentes fundamentais:

- Na forma como é concebida a intensiva construção e valor, que agora é promulgador da ação por meio criativo, intencional, integrativo e transformador das plataformas de engajamento;

- Na forma como é definida a natureza real de valor - diálogo, transparência, acessibilidade e áreas reflexivas das experiências dos stakeholders;

- Na forma como é aprofundada a fonte virtual de valor - valores emergentes dos ecossistemas de potencialidades inclusivas, geradoras, vinculadoras e evolutivas.

\section{Procedimento Metodológico}

Após identificar as dimensões do modelo DART abordadas na literatura realizou-se uma busca no questionário ISE-2017 para relacionar perguntas do relatório com cada uma das dimensões. O setor bancário brasileiro foi selecionado para a realização desta pesquisa devido ao seu elevado padrão no que se refere a desempenho e competitividade, o que faz deste um setor interessante para o desenvolvimento de estudos relacionados à análise de stakeholders (Rudzevicius et al., 2018). Além disso, a participação das empresas brasileiras do setor 
bancário na referida base de dados é mais evidente quando comparado com organizações de outros setores.

\subsection{Questionário ISE}

O índice de Sustentabilidade Empresarial BM\&F-ISE é um índice que mede o retorno médio de uma carteira teórica de ações de empresas de capital aberto e listadas na Brasil, Bolsa, Balcão (B3) com as melhores práticas de sustentabilidade. Atualmente possui 40 empresas dentre as emissoras das 200 ações mais líquidas. Adota-se uma metodologia de análise quantitativa (pontuação no questionário) e análise qualitativa (verificação dos documentos), conforme mostra a Figura 4.

Figura 4 - Método Qualitativo-Quantitativo do Questionário ISE

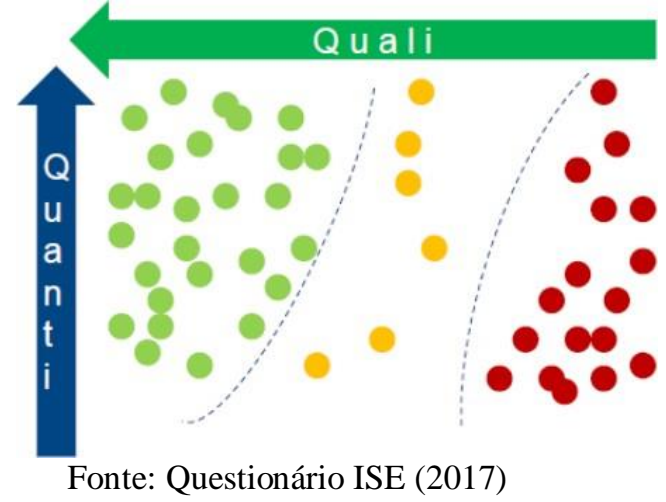

O questionário ISE é composto por 7 dimensões, sendo elas: Geral; Governança Corporativa; Natureza do Produto; Mudanças Climáticas; Ambiental; EconômicoFinanceira; Social. Dessa forma, constitui uma ferramenta para análise comparativa das empresas da B3 baseada em eficiência econômica, equilíbrio ambiental, justiça social e governança corporativa, diferenciando-as em termos de qualidade, nível de compromisso e desenvolvimento sustentável. Na figura a seguir é apresentado um exemplo de questão do ISE.

Figura 5 - Exemplo do Questionário ISE

ECO 1. As Notas Explicativas abordam de forma quantitativa:

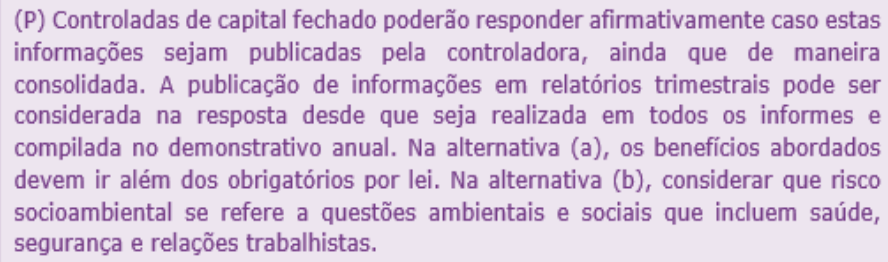

(P) Controladas de capital fechado poderão responder afirmativamente caso estas informações sejam publicadas pela controladora, ainda que de maneira consolidada. A publicação de informações em relatórios trimestrais pode ser considerada na resposta desde que seja realizada em todos os informes e compilada no demonstrativo anual. Na alternativa (a), os benefícios abordados devem ir além dos obrigatórios por lei. Na alternativa (b), considerar que risco socioambiental se refere a questões ambientais e sociais que incluem saúde, segurança e relações trabalhistas.

(GRI G4) Indicador EC1 e EC3
a) Benefícios pós-emprego
b) Gestão de Risco Corporativo Socioambiental
c) Nenhuma das anteriores

Fonte: Questionário ISE (2017) 


\subsection{Mensuração a partir das Respostas ao Questionário ISE}

Para critério de análise, foram utilizadas as respostas dos quatro bancos da carteira do questionário: Banco Bradesco S. A., Banco do Brasil S. A., Banco Santander S. A. e Itaú Unibanco Holding S. A. A escolha de análise dessas empresas foi feita baseada na representatividade em seu setor - quatro dos cinco maiores bancos na América Latina conforme observado na Figura 7 - e na possibilidade de uma análise mais detalhada referente a cada banco. A comparação também ficaria mais razoável do que utilizar os 12 setores das 30 companhias listadas no ISE, visto que alguns setores são representados somente por uma empresa, além de algumas empresas terem deixado de responder um grande número de questões.

Tabela 1 - Maiores bancos da América Latina em 2017

\begin{tabular}{|l|c|c|}
\hline \multicolumn{1}{|c|}{ Companhia } & Sede & Ativos (US\$) \\
\hline 1 - Itaú Unibanco & Brasil & 438 bilhões \\
\hline $\mathbf{2}$ - Banco do Brasil & Brasil & 430,54 bilhões \\
\hline 3 - Caixa Econômica Federal & Brasil & 385,71 bilhões \\
\hline $\mathbf{4}$ - Banco Bradesco & Brasil & 362,41 bilhões \\
\hline $\mathbf{5}$ - Banco Santander & Brasil & 215,58 bilhões \\
\hline $\mathbf{6}$ - Grupo Financeiro BBVA Bancorner & México & 100,72 bilhões \\
\hline $\mathbf{7}$ - Grupo Aval Aciones y Valores & Colômbia & 74,64 bilhões \\
\hline $\mathbf{8}$ - Grupo Financeiro Santander México SAB de CV & México & 66,69 bilhões \\
\hline 9- Bancolombia & Colômbia & 65,38 bilhões \\
\hline $\mathbf{1 0}$ - Grupo Financeiro Banamex de CV & México & 61,27 bilhões \\
\hline
\end{tabular}

Fonte: Infomoney (2017)

A primeira etapa foi uma análise de todas as perguntas do questionário, abordadas em suas 7 dimensões. Buscou-se verificar quais delas se relacionavam com elementos do modelo DART. É importante ressaltar que nas questões simbolizadas com círculos, a empresa pode assinalar somente uma resposta, enquanto em questões simbolizadas com quadrados são permitidas mais de uma resposta. Além disso, para cada questão foi realizada uma associação com os stakeholders envolvidos, divididos em colaborador, comunidade, acionista, consumidor e fornecedor. Uma mesma questão pode ser associada com mais de um stakeholder.

Para questões com alternativas apenas "sim" ou "não" foi atribuído "1" para o elemento relacionado e " 0 " no caso de resposta negativa, como no exemplo a seguir:

Figura 6 - Exemplo do Questionário ISE (2)

GER 1. $\quad$ No último ano, a companhia publicou Relatório de Sustentabilidade?
a) Sim -1.
b) Não -0.

Fonte: Questionário ISE (2017)

Para questões em que se pode assinalar mais de uma resposta, o escore do 
elemento é dado pela soma das alternativas assinaladas, de modo que a soma total tenha que chegar ao máximo de 1 ponto. Nos exemplos a seguir as alternativas não possuem qualquer tipo de hierarquia entre si.

Figura 7 - Exemplo do Questionário ISE (3)

AMB-IF 1.4. Se SIM para a PERGUNTA 1, de que maneira é feita a conscientização sobre a política corporativa?
a) Eventos ou palestras presenciais - 0,25.
b) Cursos online - 0,25.
c) Distribuição de material através de cartilhas ou outras publicações internas - 0,25.
d) Disponibilização da política na Intranet - 0,25.
e) Nenhuma das anteriores - 0 .

Fonte: Questionário ISE (2017)

Figura 8 - Exemplo do Questionário ISE (4)

GOV 40.3. Se SIM para a PERGUNTA 40, em uma ou mais das alternativas (a) a (f), o código prevê
mecanismos para a prevenção e encaminhamento de situações relacionadas às questões de compliance e
corrupção?
$\square \square$
$\square \quad$ a) Compliance - 0,5.
$\square \quad$ b) Corrupção - 0,5.

Fonte: Questionário ISE (2017)

Em outros casos, as questões podem ser hierarquizadas de acordo com suas alternativas, nas quais foi estabelecido um escore crescente e o escore total corresponde a soma das alternativas assinaladas, sempre totalizando o máximo de 1 ponto:

Figura 9 - Exemplo do Questionário ISE (5)

ECO 4.1.1. Para os tipos de risco identificados na PERGUNTA 4.1, há monitoramento periódico?

a) Sim, no mínimo anual - 0,5.

b) Sim, no mínimo semestral -1 .

c) Não -0 .

Fonte: Questionário ISE (2017) 
Figura 10 - Exemplo do Questionário ISE (6)

Assinale as alternativas que caracterizam as práticas da companhia em relação às
demonstrações financeiras:
a) Não prepara as demonstrações financeiras atualizadas monetariamente, porém
elabora $\quad 0,25$.
relatórios gerenciais atualizados monetariamente para uso interno - Prepara as demonstrações financeiras atualizadas monetariamente, porém as mesmas não são
publicadas - 0,5.
c) Prepara e publica as demonstrações financeiras atualizadas monetariamente - 1.

Fonte: Questionário ISE (2017)

Algumas questões foram associadas a mais de um elemento. Nesse caso, desconsiderou-se a questão para análise. A tabela a seguir mostra a relação de questões encontradas para cada elemento do modelo DART.

Tabela 2 - Quantidade de questões por elemento do modelo DART

$\begin{array}{lc}\text { Elemento } & \frac{\text { Questões }}{101} \\ \text { Risco } & 55 \\ \text { Transparência } & 28 \\ \text { Acesso } & \underline{35} \\ \text { Diálogo } & \mathbf{2 1 9} \\ \text { Total } & \text { Fonte: Os autores } \\ & (2020)\end{array}$

A quantidade de questões encontradas para cada stakeholder observado encontrase na tabela seguinte.

\begin{tabular}{cc}
$\begin{array}{c}\text { Tabela } 3 \text { - Total de questões por Stakeholder } \\
\text { Stakeholder }\end{array}$ & $\begin{array}{c}\text { Questões } \\
\text { Comunidade }\end{array}$ \\
Colaboradores & 8311 \\
Consumidores & 50 \\
Acionistas & 41 \\
Fornecedores & $\underline{30}$ \\
\hline Total Fonte: Os autores (2020) & $\mathbf{3 1 5}$
\end{tabular}

Essas questões foram contabilizadas em um banco de dados no software Excel de acordo com o número da pergunta, a dimensão, o elemento associado, o stakeholder envolvido e a pontuação referente a cada um dos quatro bancos analisados, seguindo o modelo apresentado na Tabela 4. 
Tabela 4 - Coleta de dados da base ISE

\begin{tabular}{|c|c|c|c|c|c|c|c|c|c|c|c|c|}
\hline 3 & Pergunta & Dimensão & Elemento & Stakeholder 1 & Stakeholder 2 & Stakeholder 3 & Stakeholder 4 & Stakeholder 5 & $\begin{array}{l}\text { B } \\
\text { C } \\
\text { O } \\
\\
\text { B } \\
\text { R } \\
\text { A } \\
\text { D } \\
\text { E } \\
\text { S } \\
\text { C } \\
\text { O }\end{array}$ & $\begin{array}{l}\text { B } \\
\text { C } \\
\text { O } \\
\\
\text { B } \\
\text { R } \\
\text { A } \\
\text { S } \\
\text { I } \\
\text { L }\end{array}$ & $\begin{array}{l}\text { B } \\
\text { C } \\
\mathrm{O} \\
\\
\mathrm{S} \\
\mathrm{A} \\
\mathrm{N} \\
\mathrm{T} \\
\mathrm{A} \\
\mathrm{N} \\
\mathrm{D} \\
\mathrm{E} \\
\mathrm{R}\end{array}$ & $\begin{array}{l}\text { I } \\
\mathrm{T} \\
\mathrm{A} \\
\mathrm{U} \\
\mathrm{U} \\
\mathrm{N} \\
\mathrm{I} \\
\mathrm{B} \\
\mathrm{A} \\
\mathrm{N} \\
\mathrm{C} \\
\mathrm{O}\end{array}$ \\
\hline 4 & AMB-IF 1 & Ambiental IF & Risco & Comunidade & & & & & 1,00 & 1,00 & 1,00 & 1,00 \\
\hline 5 & AMB-IF 1.1 & Ambiental IF & Risco & Comunidade & & & & & 0,75 & 1,00 & 1,00 & 1,00 \\
\hline 6 & AMB-IF 1.2 & Ambiental IF & Risco & Comunidade & & & & & 0,80 & 1,00 & 0,64 & 1,00 \\
\hline 7 & AMB-IF 1.3 & Ambiental IF & Dialogo & Colaborador & & & & & 1,00 & 1,00 & 1,00 & 1,00 \\
\hline 8 & AMB-IF 1.4 & Ambiental IF & Dialogo & Colaborador & & & & & 1,00 & 1,00 & 1,00 & 1,00 \\
\hline 9 & AMB-IF 18 & Ambiental IF & Risco & Consumidor & & & & & 1,00 & 1,00 & 1,00 & 1,00 \\
\hline 10 & AMB-IF 18.2 & Ambiental IF & Transparencia & Consumidor & & & & & 1,00 & 1,00 & 0,50 & 1,00 \\
\hline 11. & AMB-IF 19 & Ambiental IF & Risco & Consumidor & & & & & 1,00 & 0,60 & 0,80 & 1,00 \\
\hline 12 & AMB-IF 20 & Ambiental IF & Risco & Comunidade & & & & & 1,00 & 1,00 & 1,00 & 1,00 \\
\hline 13 & AMB-IF 20.1 & Ambiental IF & Risco & Comunidade & & & & & 0,80 & 1,00 & 0,85 & 0,85 \\
\hline 14 & AMB-IF 20.2 & Ambiental IF & Risco & Comunidade & & & & & 1,00 & 1,00 & 0,66 & 0,77 \\
\hline 15 & AMB-IF 20.3 & Ambiental IF & Transparencia & Comunidade & & & & & 1,00 & 1,00 & 1,00 & 1,00 \\
\hline 16 & AMB-IF 22 & Ambiental IF & Risco & Colaborador & & & & & 1,00 & 1,00 & 1,00 & 0,25 \\
\hline 17 & AMB-IF 23 & Ambiental IF & Risco & Comunidade & & & & & 1,00 & 1,00 & 1,00 & 1,00 \\
\hline 18 & AMB-IF 24 & Ambiental IF & Risco & Comunidade & & & & & 1,00 & 0,25 & 0,75 & 1,00 \\
\hline 19 . & ECO 1 & Econômico-Financeira & Risco & Colaborador & & & & & 0,50 & 0,50 & 0,50 & 1,00 \\
\hline 20 & ECO 1.1 & Econômico-Financeira & Risco & Comunidade & & & & & 0,00 & 1,00 & 1,00 & 1,00 \\
\hline
\end{tabular}

Fonte: Os autores (2020)

\section{Resultados da Pesquisa}

Os resultados obtidos a partir da coleta de dados realizada são apresentados neste tópico que possui o objetivo de apresentar uma análise comparativa dos indicadores referentes aos quatro bancos, de modo a permitir a visualização do melhor e pior desempenho, além de dados estatísticos como média, mediana, mínimo, máximo e desvio padrão tanto para os elementos do modelo DART quanto para cada stakeholder. A partir de tal comparação, torna-se possível mensurar a distribuição dos elementos em sua nota total e o desempenho relacionado aos cinco stakeholders observados.

\subsection{Análise Comparativa}

A análise de dados iniciou-se com a descrição dos valores dos rankings por elemento e por stakeholder, conforme Tabela 5 e Tabela 6:

Tabela 5 - Média, mediana e desvio padrão por elemento do DART

\begin{tabular}{lccccc} 
Elemento & Média & Desvio Padrão & Mediana & Mínimo & Máximo \\
\hline Acesso & 0,653 & 0,055 & 0,653 & 0,601 & 0,707 \\
Diálogo & 0,906 & 0,058 & 0,896 & 0,857 & 0,976 \\
Risco & 0,833 & 0,040 & 0,824 & 0,798 & 0,887 \\
Transparência & 0,824 & 0,031 & 0,825 & 0,789 & 0,857 \\
\hline Geral & 0,804 & 0,010 & 0,800 & 0,761 & 0,857
\end{tabular}

Fonte: Os autores (2020) 
Tabela 6 - Média, mediana e desvio padrão por stakeholder

\begin{tabular}{lccccc} 
Elemento & Média & Desvio Padrão & Mediana & Mínimo & Máximo \\
\hline Acionistas & 0,895 & 0,015 & 0,896 & 0,878 & 0,912 \\
Colaboradores & 0,816 & 0,026 & 0,815 & 0,786 & 0,847 \\
Comunidade & 0,878 & 0,038 & 0,870 & 0,842 & 0,931 \\
Consumidores & 0,834 & 0,057 & 0,829 & 0,781 & 0,898 \\
Fornecedores & 0,905 & 0,035 & 0,894 & 0,877 & 0,954 \\
\hline Geral & 0,866 & 0,011 & 0,861 & 0,833 & 0,908 \\
\hline
\end{tabular}

Fonte: Os autores (2020)

Por meio da análise da Tabela5, pode-se perceber que o elemento "acesso" possui a menor média $(0,653)$, enquanto o elemento "diálogo" se destaca com média de 0,906 . A nota máxima do "diálogo" é muito superior as demais $(0,976)$. Vale ressaltar que a média do acesso é muito inferior aos demais elementos, o que revela uma grande necessidade de avanço dos bancos envolvidos nesse ramo. Com relação ao desvio padrão, nota-se o maior desvio para o elemento "diálogo", muito em razão do alto desempenho por parte do banco ITAÚ UNIBANCO HOLDING S.A., como se verá na análise individual. No entanto, na média o desvio não se mostrou elevado.

$\mathrm{Na}$ Tabela 6 destaca-se o desempenho elevado do stakeholder fornecedores, obtendo a maior média da amostra $(0,905)$. O desvio padrão dos consumidores variou de forma relevante em relação ao restante $(0,057)$. A média dos máximos de cada stakeholder $(0,908)$ pode ser considerada elevada, o que revela um destaque para as notas mais altas em cada um.

A Tabela 7 e o Gráfico 1, ambos apresentados a seguir, permitem uma análise comparativa por elemento: No "acesso", o banco SANTANDER S. A. obteve menor nota $(0,601)$ e o banco ITAÚ HOLDING S. A. se destacou com a nota de 0,707. Ainda assim, os quatro bancos obtiveram uma nota baixa nesse elemento se comparado aos outros três. No "diálogo", destaque para o BANCO DO BRASIL S. A $(0,931)$ e o banco ITAÚ HOLDING S. A. (0,976). O elemento "risco" apresentou as maiores notas nos bancos ITAÚ UNIBANCO HOLDING S. A. $(0,887)$ e SANTANDER S. A. $(0,841)$. Por fim, no âmbito da "transparência" a maior nota não se destacou muito em relação aos demais, sendo a primeira 0,857 e a segunda 0,841 . Vale ressaltar que o banco ITAÚ UNIBANCO HOLDING S. A. demonstrou desempenho superior aos demais em todos elementos com exceção da "transparência".

Tabela 7 - Comparação por elemento do modelo DART nos bancos

\begin{tabular}{ccccc} 
Elemento & BCO BRADESCO & BCODOBRASIL & BCO SANTANDER & ITAUUNIBANCOHOLDING \\
& S.A. & S.A. & S.A. & S.A. \\
\hline Acesso & 0,612 & 0,694 & 0,601 & 0,707 \\
Diálogo & 0,861 & 0,931 & 0,857 & 0,976 \\
Risco & 0,798 & 0,807 & 0,841 & 0,887 \\
Transparência & 0,841 & 0,808 & 0,789 & 0,857 \\
\hline
\end{tabular}

Fonte: Os autores (2020) 


\section{Gráfico 1 - Comparação por elemento do modelo DART nos bancos}

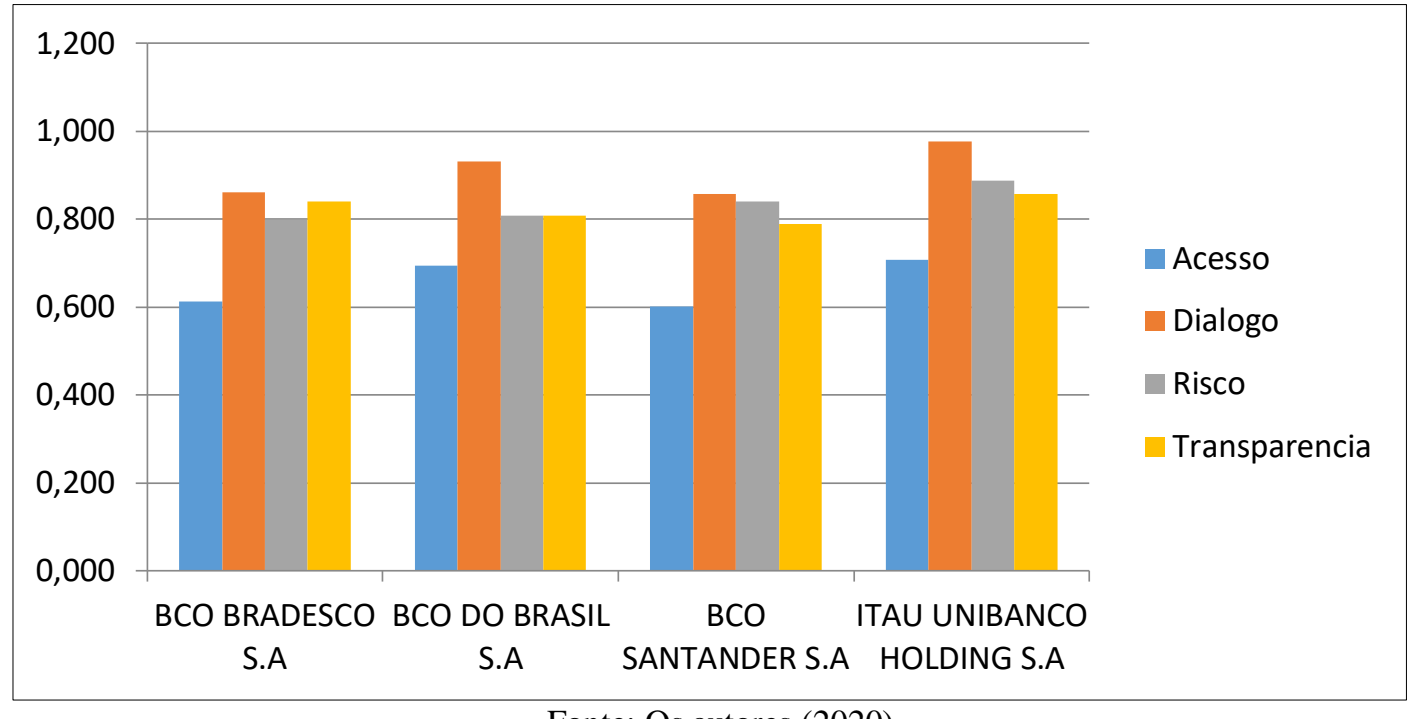

Fonte: Os autores (2020)

Finalmente, por meio da observação da Tabela 8 e do Gráfico 2 abaixo, pode-se elaborar o seguinte ranking final de cocriação para os bancos estudados: $1^{\circ}$ BANCO ITAÚ HOLDING S. A. $(0,857) ; 2^{\circ}$ BANCO DO BRASIL S. A. $(0,810) ; 3^{\circ}$ BANCO BRADESCO S. A. $(0,778)$; e $4^{\circ}$ BANCO SANTANDER S. A. $(0,772)$.

Tabela 8 - Nota geral no modelo DART dos bancos estudados

\begin{tabular}{cc} 
Banco & Nota final \\
\hline BCO BRADESCO S. A. & $\mathbf{0 , 7 7 8}$ \\
BCO DO BRASIL S. A. & $\mathbf{0 , 8 1 0}$ \\
BCO SANTANDER S. A. & $\mathbf{0 , 7 7 2}$ \\
ITAU UNIBANCO HOLDING S. A. & $\underline{\mathbf{0 , 8 5 7}}$ \\
\hline \multicolumn{2}{c}{ Fonte: Os autores (2020) }
\end{tabular}

Gráfico 2 - Nota geral no modelo DART dos bancos estudados

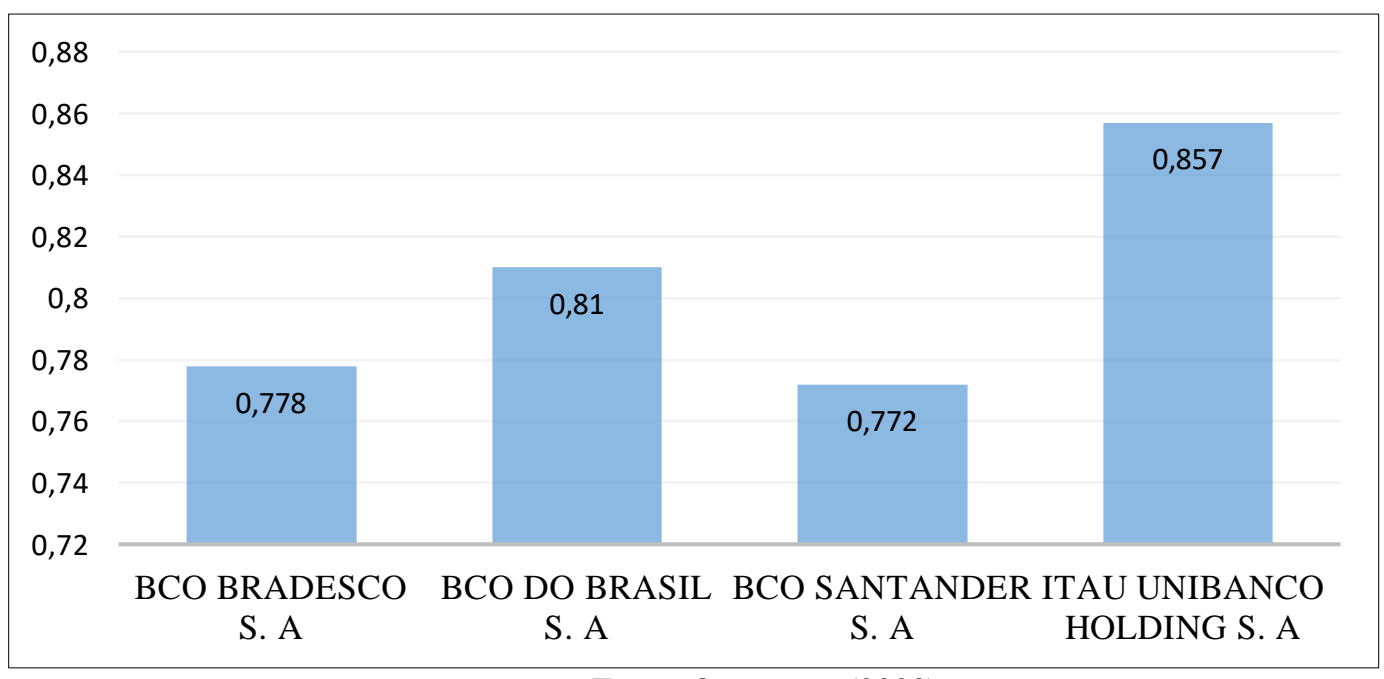

Fonte: Os autores (2020) 
Observa-se, dessa forma, que a nota geral do primeiro banco é bastante superior aos demais, enquanto as notas dos dois últimos são próximas, diferenciando-se apenas na última casa decimal.

\subsection{Discussão dos Resultados}

Com base nas abordagens de Prahalad e Ramaswamy (2004) e acompanhando atualizações como as de Ramaswamy e Ozcan (2014), Johns e Davey (2019), entre outros, foi possível observar empiricamente o comportamento das quatro variáveis do modelo DART na ideia da cocriação no setor bancário brasileiro. Esta pesquisa corrobora com estudos anteriores que argumentam acerca da comunicação - ou diálogo - como relevante elemento gerencial capaz de incentivar aprendizagem mútua entre grupos de interesse e desenvolvimento em suas relações (Raelin, 2013; Chakraborty, \& Dobrzykowski, 2014).

Nessa perspectiva, este estudo revelou que o elemento "diálogo" possui uma relevância mais definida para os bancos analisados, possuindo maior influência na elucidação da nota final de cada empresa no referido modelo. Considerando as informações obtidas, entende-se que, no referido setor, o desenvolvimento de canais de comunicação pode aprimorar o relacionamento entre empresas e seus stakeholders, possivelmente influenciando uma maior satisfação geral por parte das partes interessadas. Organizações que fornecem espaço para diálogo e buscam sugestões de seus grupos de interesse podem apresentar produtos e serviços mais competitivos e inovadores, ampliando suas possibilidades de crescimento e influência no mercado.

Dessa forma, argumentamos que o desenvolvimento de canais de comunicação, incentivando o diálogo entre as partes, reduz potenciais conflitos e ambiguidades e fortalece fatores importantes, como a confiança na relação entre empresa e stakeholders. Logo, compreende-se que, por meio do diálogo as potenciais mudanças nas demandas do mercado podem ser bem atendidas, bem como as percepções e expectativas dos stakeholders podem ser consideradas.

Conforme observado e considerando as demais variáveis, outros elementos, como a "transparência" e o "acesso", apesar de ser menos considerado nos bancos analisados, também podem influenciar no desenvolvimento da confiança entre empresas e partes interessadas. Estes elementos podem, por exemplo, servir de insumo para o elemento "risco", ampliando a segurança na realização de análises de riscos, atividade fundamental para a sustentabilidade empresarial no setor estudado, bem como podem influenciar no elemento diálogo, conforme pode ser observado em trabalhos anteriores (Oliveira et al., 2014) onde é possível observara importância da transparência no relacionamento e comunicação com os stakeholders.

\section{Considerações Finais}

Esta pesquisa visou analisar e mensurar o processo de cocriação de valor no setor bancário brasileiro com a utilização de dados oriundos do Questionário ISE-2017 que se relacionavam com os elementos de diálogo, acesso, risco e transparência, observados no modelo DART (Prahalad, \& Ramaswamy, 2004). Para tanto, realizou-se previamente uma pesquisa bibliográfica sobre a literatura pertinente ao tema objeto da pesquisa e assuntos correlatos - como o conceito de valor, criação de valor e cocriação de valor. Nessa etapa buscou-se responder a questões centrais relacionadas ao tema: O que é valor? Quais são as diferentes definições de valor? Como funciona o processo tradicional de criação de valor? $\mathrm{O}$ que diferencia o processo tradicional para o processo de cocriação de valor? Como evoluiu 
esse processo ao longo do tempo? O que é o modelo DART e como ele é operacionalizado? Como se extrapola o conceito de cocriação para outros stakeholders além dos clientes? As perguntas foram respondidas durante a pesquisa bibliográfica e complementadas com a pesquisa empírica.

$\mathrm{Na}$ etapa empírica, foi possível observar que as empresas analisadas ainda precisam de desenvolvimento na questão do "acesso". A nota geral do elemento foi muito inferior as demais. Em questões como "Qual a porcentagem de negros em cargos de gerência?" ou "Qual a porcentagem de mulheres em cargos de gerência?" todas as empresas apresentaram baixo desempenho. Dessa forma, a ampliação do aprendizado desse conceito mostra-se relevante nesse contexto. Também se observou que os elementos "diálogo" e "transparência" possuem as notas mais elevadas, possivelmente por ser uma relação com aplicações já de conhecimento geral, como participações de empresas em fóruns de consumidores e divulgação de informações nos sítios eletrônicos das empresas. Os elementos, portanto, não se encontravam bem distribuídos no momento da pesquisa. É importante mencionar também que o BANCO ITAÚ UNIBANCO HOLDING S. A. apresentou as maiores notas nos quatro elementos.

Esse estudo encontrou algumas limitações que devem ser sinalizadas para que possam ser superadas em pesquisas futuras. Primeiro, o processo de cocriação ainda pode ser considerado novo e não há uma bibliografia extensa e variada sobre o tema. Os autores referência se repetem constantemente, variando somente o ano de publicação de artigos. Segundo, a quantidade reduzida de empresas no formulário não permitiu uma análise por setores de atuação, uma vez que alguns setores eram representados somente por uma ou duas empresas.

Além disso, havia um formulário com questões específicas para empresas do setor bancário, o que dificultava uma análise de todas as empresas juntas. Terceiro, o modo de mensuração não é completamente assertivo, por exemplo: uma alternativa que vale 0,5 não possui necessariamente duas vezes mais valor de cocriação do que uma alternativa que vale 0,25. No entanto, esse modo foi o mais próximo encontrado para mensurar esse processo novo e ainda pouco utilizado. Por fim, como foi feita a análise de somente um ano do questionário (2017/2018), não foi possível a realização de uma análise de correlação entre variáveis independentes - diálogo, transparência, risco e acesso; e a variável dependente nota final de cocriação.

Algumas recomendações de pesquisa podem então ser apontadas, como uma análise comparativa entre diferentes setores da indústria. Outra possibilidade de estudo seria a realização de uma análise detalhada de questões de cada um dos elementos para revelar, por exemplo, quais stakeholders são priorizados, de fato, nos processos de cocriação em empresas de indústrias específicas.

\section{Referências Bibliográficas}

Alves, H., Fernandes, C., \& Raposo, M. (2016). Value co-creation: Concept and contexts of application and study. Journal of Business Research, 69(5), 1626-1633.

Aurier, P., Evrard, Y., \& N'Goala, G. (1998). La valeur du produit du point de vue du consommateur. Actes des XI Vèmejournées Nationales des IAE, Nantes, 15-26. 
B3 (2017). Índice de Sustentabilidade Empresarial. Disponível em: <https://www.isebvmf.com.br/>. Acesso em: julho de 2018.

Chakraborty, S., \& Dobrzykowski, D. (2014). Examining value co-creation in healthcare purchasing: A supply chain view. Business: Theory and Practice, 15(2), 179-190.

Choi, H., \& Burnes, B. (2013). The internet and value co-creation: the case of the popular music industry. Prometheus, 31(1), 35-53.

Dodds, W. B., Monroe, K. B., \& Grewal, D. (1991). Effects of price, brand, and store information on buyers' product evaluations. Journal of Marketing Research, 307-319.

Franco, D. A. (2012). Cocriação: reinventando o conceito. Festival de Ideias, São Paulo.

Gale, B. T., \& Wood, R. C. (1994). Managing customer value: Creating quality and services that customers can see. New York.

Holbrook, M. B. (1994). The nature of customer value: an axiology of services in the consumption experience. Service Quality: New Directions in Theory and Practice, 21, 21-71.

Holbrook, M. B. (1999). Consumer value: a framework for analysis and research. Psychology Press.

Holbrook, M. B., \& Hirschman, E. C. (1982). The experiential aspects of consumption: Consumer fantasies, feelings, and fun. Journal of Consumer Research, 9(2), 132-140.

Ind, N., \& Coates, N. (2013). The meanings of co-creation. European Business Review, 25(1), 86-95.

Johns, R., \& Davey, J. (2019). Introducing the transformative service mediator: Value creation with vulnerable consumers. Journal of Services Marketing.

Kujala, J., Lehtimäki, H., \& Myllykangas, P. (2017). Value Co-creation in Stakeholder Relationships: A Case Study. In Stakeholder Engagement: Clinical Research Cases (15-30). Springer, Cham.

Mangini, E. R., Rossini, F. H. B., Conejero, M. A., \& Urdan, A. T. (2018). Gestão do Conhecimento como Fator de Alavancagem de Inovação Aberta: o caso Foco Virtual. Revista de Administração, Sociedade e Inovação, 4(1), 16-33.

Monroe, K. B. (1990). Pricing: Making profitable decisions. McGraw-Hill Companies.

Oliveira, G., Damacena, C., \& Brambilla, F. R. (2014). Cocriação como proposta para geração de valor: caso da empresa dental américa. Desenvolve Revista de Gestão do Unilasalle, 3(2), 129-150. 
Overby, J. W. (2001). The impact of national culture upon the customer value hierarchy: A comparison between French and American consumers.

Parmar, B. L., Freeman, R. E., Harrison, J. S., Wicks, A., Purnell, L., \& De Colle, S. (2010). Stakeholder Theory: The State of the Art. Academy of Management Annals, 4, 403445.

Pater, M. (2009). Co-creation's five guiding principles. Fronteer Strategy.

Payne, A., \& Frow, P. (2005). A strategic framework for customer relationship management. Journal of Marketing, 69(4), 167-176.

Prahalad, C. K., \& Ramaswamy, V. (2000). Co-opting customer competence. Harvard Business Review, 78(1), 79-90.

Prahalad, C. K., \& Ramaswamy, V. (2004). Co-creating unique value with customers. Strategy \& Leadership, 32(3), 4-9.

Prahalad, C. K., \& Ramaswamy, V. (2004). The future of competition: Co-creating unique value with customers. Harvard Business Press.

Raelin, J. A. (2013). The manager as facilitator of dialogue. Organization, 20(6), 818839.

Ramaswamy, V., \& Gouillart, F. (2010). Building the co-creative enterprise. Harvard Business Review, 88(10), 100-109.

Ramaswamy, V., \& Gouillart, F. J. (2010). The power of co-creation: Build it with them to Boost Growth, Productivity, and Profits. Simon and Schuster.

Ramaswamy, V., \& Ozcan, K. (2014). The co-creation paradigm. Stanford University Press.

Randall, W. S., Gravier, M. J., \& Prybutok, V. R. (2011). Connection, trust, and commitment: dimensions of co-creation? Journal of Strategic Marketing, 19(01), 3-24.

Ranjan, K., Read, S. (2014). Value co-creation: concept and measurement. Journal of the Academy of Marketing Science, p. 1-26.

Ribeiro, H. C. M., \& Costa, B. K. (2016). Cocriação de valor: uma bibliometria de 2000 a 2014. Revista Eletrônica de Estratégia \& Negócios, 9(1), 118-151.

Richins, M. L. (1994). Valuing things: The public and private meanings of possessions. Journal of Consumer Research, 21(3), 504-521.

Rokeach, M. (1973). The nature of human values. Freepress. 
Rudzevicius, R. V., Boaventura, J. M. G., Mascena, K. M. C., \& Sarturi, G. (2018). Alocação de Valor para os Stakeholders: Um Estudo no Setor Financeiro. Revista de Administração da Unimep, 16(1), 56-77.

Sanders, E. B. N., \& Stappers, P. J. (2008). Co-creation and the new landscapes of design. Co-Design, 4(1), 5-18.

Silva, S. B., \& Villan, W. J. (2018). O Papel da Coopetição na Criação de Valor para Micro e Pequenas Empresas-MPEs no Âmbito da Cadeia de Suprimentos em Relações Fornecedor-Fornecedor. Revista de Administração, Sociedade e Inovação, 4(1), 90-105.

Szybillo, G. J., \& Jacoby, J. (1974). Intrinsic versus extrinsic cues as determinants of perceived product quality. Journal of Applied Psychology, 59(1), 74.

Tantalo, C., \& Priem, R. L. (2016). Value creation through stakeholder synergy. Strategic Management Journal, 37(2), 314-329.

Thaler, R. (1985). Mental accounting and consumer choice. Marketing Science, 4(3), $199-214$.

Vargo, S. L., \& Lusch, R. F. (2004). Evolving to a new dominant logic for marketing. Journal of Marketing, 68(1), 1-17.

Vargo, S. L., Maglio, P. P., \& Akaka, M. A. (2008). On value and value co-creation: a service systems and service logic perspective. European Management Journal, 26, 3, pp. 145152

Von Hippel, E. (1986). Lead users: a source of novel product concepts. Management Science, 32(7), 791-805.

Woodruff, R. B., \& Gardial, S. (1996). Know your customer: New approaches to understanding customer value and satisfaction. Wiley.

Zeithaml, V. A. (1988). Consumer perceptions of price, quality, and value: a meansend model and synthesis of evidence. The Journal of Marketing, 2-22.

Zwass, V. (2010). Co-creation: Toward a taxonomy and an integrated research perspective. International Journal of Electronic Commerce, 15(1), 11-48. 